Geopolítica(s) Revista de estudios sobre espacio y poder ISSN: 2172-3958

\title{
Turismo comunitario en territorios conflictivos. El caso de las comunidades indígenas mapuche en la Región de los Ríos en Chile ${ }^{1}$
}

\author{
Marisela Jeanette Pilquimán Vera²
}

Recibido: 19 de junio de 2015 / Aceptado: 31 de diciembre de 2016

Resumen. La recuperación del territorio ancestral, el fortalecimiento de la cultura y la revalorización de los conocimientos propios son actualmente prioridades de los pueblos originarios en Chile. Es el caso de las comunidades mapuche en la comuna de Panguipulli, Región de Los Ríos en el sur de Chile, donde el turismo comunitario se ha posicionado, desde el imaginario indígena, en una alternativa posible para reivindicar estos objetivos frente a la sociedad nacional dominante hegemónica y como una estrategia emergente en la que convergen la resistencia, la revalorización de prácticas culturales y la generación de oportunidades económicas. En este artículo, a partir de un abordaje metodológico de investigación cualitativa complementada con métodos y técnicas cuantitativos, se explora en los imaginarios y las experiencias de estas comunidades originarias para revelar aspectos que contribuyan a la construcción de un modelo de desarrollo turístico pertinente en territorios en conflicto que progresivamente reflejan los efectos de la globalización.

Palabras clave: turismo; territorio; comunidades mapuche; turismo comunitario; Chile.

\section{[en] Community Tourism in Conflict Territories. The Case of Mapuche Indigenous Communities in the Los Ríos Region in Chile}

\begin{abstract}
For present-day indigenous populations in Chile, the recovery of ancestral lands, the strengthening of indigenous culture and the rediscovery of traditional knowledge are priority matters. This is certainly the case for the Mapuche communities of the Panguipulli municipality, located in the Los Ríos Region. In the indigenous imagery, community tourism has become a powerful instrument for the presentation of demands before the hegemonic national society. An emergent strategy, community tourism brings together the possibility to organize resistance against domination practices, reengage indigenous cultural practices and generate economic opportunities. This paper adopts a qualitative methodological approach, but uses quantitative methods and techniques as well. The imagery and experiences of indigenous communities are explored in order to unearth the elements that contribute to the construction of a tourism development pattern suitable for conflict territories where the effects of globalization become visible by the day.
\end{abstract}

Keywords: tourism; territory; Mapuche communities; community tourism; Chile.

1 La autora de este artículo desea agradecer a los integrantes de las comunidades mapuche ancestrales Juan Caripán, Juan Chañapi, Ramón Chincolef y Emilio Epuñanco. También a las comunidades indígenas mapuche Inalafken, Cacique Llancapán y a la Asociación de Pequeños Agricultores y Artesanos de Pucura por sus valiosos aportes para la realización de este estudio.

2 Centro de Estudios del Desarrollo Regional y Políticas Públicas, Universidad de Los Lagos (Chile)

E-mail: marisela.pilquiman@ulagos.cl 


\title{
[pt] Turismo comunitário em territórios conflitivos. O caso das comunidades indígenas mapuche na Región de los Ríos no Chile
}

\begin{abstract}
Resumo. A recuperação do território ancestral, o fortalecimento da cultura e a revalorização dos conhecimentos próprios são atualmente prioridades dos povos originários no Chile. Este é o caso das comunidades mapuche no município de Panguipulli, Región de Los Rios no sul do Chile, onde o turismo comunitário posicionou-se, a partir do imaginário indígena, como uma possível alternativa para reivindicar esses objetivos contra a sociedade nacional dominante e hegemônica, e como uma estratégia emergente na qual convergem resistência, valorização das práticas culturais e geração de oportunidades econômicas. Este artigo, por meio de abordagem metodológico qualitativo complementado por técnicas e métodos quantitativos, explora o imaginário e as experiências dessas comunidades indígenas para revelar aspectos que contribuem para a construção de um modelo de desenvolvimento turístico pertinente em territórios em conflito, que progressivamente refletem os efeitos da globalização.
\end{abstract}

Palavras-chave: turismo; território; comunidades mapuche; turismo comunitário; Chile.

Sumario. Introducción. 1. El contexto regional. 2. Metodología. 3. Análisis y resultados. 3.1. Expansión turística en territorios conflictivos. 3.2. Entre turismo masivo y turismo comunitario. 3.3. Encuentros y desencuentros entre comunidades anfitrionas y visitantes. Conclusiones. Bibliografía.

Cómo citar: Pilquimán Vera, Marisela Jeanette (2017) “Turismo comunitario en territorios conflictivos. El caso de las comunidades indígenas mapuche en la Región de los Ríos en Chile”. Geopolítica(s). Revista de estudios sobre espacio y poder, vol. 8, núm. 1, 11-28.

\section{Introducción}

El turismo se ha constituido en uno de los fenómenos económicos, sociales y culturales más importante acontecidos desde el siglo pasado hasta el presente; según la Organización Mundial del Turismo (OMT, 2015), de ser una práctica que a inicios del siglo XX era propia de un segmento exclusivo de la sociedad, en las seis últimas décadas ha experimentado una continua expansión y diversificación al convertirse en una necesidad social vinculada a los períodos de descanso (Bustos, 2005) que se espera continúe en aumento en correspondencia con las positivas tendencias que lo sitúan como una actividad económica en crecimiento a nivel mundial (OMT, 2015).

Chile no constituye una excepción a esta tendencia debido al peso superlativo que el turismo ha adquirido en el concierto político y económico como uno de los sectores más dinámicos y de mayor crecimiento del país. Este posicionamiento ha resultado de la convergencia de una serie de factores que van desde la existencia de recursos naturales y culturales de particular calidad, una economía abierta a la inversión extranjera, la favorable imagen de democracia y estabilidad del país ante la comunidad internacional, el reimpulso de las políticas de inversión en obras de infraestructura, especialmente vial y aeroportuaria, un adecuado nivel de seguridad, escasos requerimientos de visado, hasta la liberación de mayor tiempo para el ocio y su aprovechamiento efectivo (Bustos, 2005; Sepúlveda, 2001), situación que ha implicado no sólo el aumento de las migraciones turísticas, sino también la diversificación de la oferta, y en consecuencia, la incorporación de territorios rurales donde hasta hace pocos años la actividad turística no se desarrollaba (Bustos, 2005). 
De esta forma, la diversificación productiva del espacio rural se ha manifestado, también, con el surgimiento de una serie de iniciativas de turismo vinculadas a modalidades no convencionales (Schaerer \& Dirven, 2001) que permiten al viajero compenetrarse con formas de vida propias de sujetos sociales que han descubierto en esta actividad una alternativa para enfrentar la crisis estructural de la agricultura tradicional. Así, en el marco de la nueva ruralidad, el surgimiento de estos nuevos turismos en diversas modalidades - tales como las de turismo rural, agroturismo, turismo ecológico o ecoturismo, etnoturismo, turismo comunitario, entre otras- ha sido fundamentalmente posible por efecto de las políticas públicas destinadas a dinamizar territorios rurales, junto a una serie de acciones provenientes de organizaciones no gubernamentales, del mundo académico, e incluso nacidas de motu proprio desde las propias comunidades rurales e indígenas, que llegan a considerar que el turismo - en sus diversas modalidades - es una opción para mitigar los problemas presentes en el mundo rural y, en especial, de desarrollo para los pueblos originarios, aprovechando el creciente interés que despierta entre los visitantes, la naturaleza, la cultura y las relaciones con las etnias locales (Bustos, 2005).

Tal es el caso del pueblo mapuche ("gente de la tierra", en su lengua, el mapuzüngun), constituida actualmente en la sociedad indígena mayoritaria del país (INE, 2002) que históricamente habita desde tiempos precolombinos parte de los territorios de lo que hoy constituye el Estado de Chile (véase Bengoa, 1985) para quienes la posibilidad de atraer el flujo turístico - y, por esta vía, no sólo generar ingresos sino, eventualmente, revalorizar su cultura - es una consideración de importancia (Pilquimán \& Skewes, 2010).

Sin embargo, para los mapuche la actividad turística ha trascendido a su rol habitual de agente dinamizador de la economía local al desenvolverse en un especial ambiente de reivindicación territorial, cultural y de autonomía fundado básicamente con la reducción drástica de su territorio histórico ${ }^{3}$, incluso el reconocido a través de Títulos de Merced ${ }^{4}$ (Toledo, 2006).

Todo lo anterior acompañado por un proceso de aculturación y/o asimilación cultural, la exigencia de reconocimiento y respeto de sus derechos (políticos, territoriales, culturales, económicos y sociales) como pueblo (Toledo, 2006) y sus actuales aspiraciones de desarrollar planes de vida destinados a mitigar los efectos perniciosos de las políticas de orientación neoliberal y a promover sus propias visiones de bienestar en los territorios que habitan. Y a esto se suma, además, la pa-

3 La pérdida del territorio del pueblo mapuche iniciado con la conquista española, en 1540, con diversos bemoles hasta mediados del siglo XIX, había demostrado mantener para este pueblo un cierto grado de autonomía territorial reconocido por la Corona española y más tarde por el Estado chileno que progresivamente inició un proceso de ocupación militar denominado “Pacificación de la Araucanía” llevado a cabo entre 1860 y 1881 (véase Toledo, 2006; Bengoa, 1985; Mariman, 2006). Luego, en 1883, el Estado comienza un periodo de despojo territorial mediante un proceso de Radicación de la población mapuche, consistente en relocalizar en reducciones, esto es, pequeñas extensiones de tierra de dominio común registradas bajo Títulos de Merced. Posteriormente, desde 1927, los territorios de estas comunidades fueron divididos y entregados en títulos individuales como parte de una nueva política de desarrollo agro-exportador. Cobra importancia este suceso toda vez que las actuales reivindicaciones de tierras hacen referencia a estos Títulos de Merced (Viera, 2013).

4 Estos títulos se entregaron en virtud de la Ley del 4 de diciembre de 1866 por la Comisión Radicadora de Indígenas, en las provincias de Bio Bio, Arauco, Malleco, Cautín, Valdivia y Osorno, iniciándose la titulación en 1884 y terminando el proceso en el año 1929. Entre las regiones VIII, IX y X se otorgaron 2.918 Títulos de Merced, con una superficie total de 510.386,67 ha. En la actualidad un porcentaje de estas tierras se encuentra perdido y/o usurpado mediante diversos mecanismos legales e ilegales (Comisión Verdad Histórica y Nuevo Trato con los Pueblos Indígenas, 2008). 
radójica relación que sostiene este pueblo originario con el Estado chileno, que mientras le reconoce algunos derechos, le niega otros ${ }^{5}$.

En este contexto, el turismo comunitario se presenta como una alternativa al modelo de turismo convencional (Guzmán-López et al., 2011). Puede ser conceptualizado como una forma de gestión del turismo que busca equilibrar las dimensiones medioambientales y las culturales, con la particularidad de una gestión y organización anclada en las comunidades (Ruiz \& Solís, 2007) que, además de ofrecer a los visitantes los atractivos que en el territorio se ubican, les brinde una posibilidad para experimentar la vida local (Sampaio \& Zamigan, 2012; Grimm \& Sampaio, 2011; Solís, 2007). Se caracteriza por ser un "turismo de empoderamiento y concientización" en especial para sus oferentes. Este turismo, al cruzarse con los actuales procesos reivindicativos de las comunidades mapuche, se ha posicionado en el imaginario social indígena —al igual que en otras comunidades originarias en Latinoamérica (véase FEPTC, citado por Solís, 2007; Bohórquez et al., 2015; Coca, 2012; Cox, 2012) - como una opción para generar renta y empleo, además de una estrategia emergente para revalorizar su cultura frente a la sociedad nacional dominante hegemónica que pone en relieve su proceso reivindicativo (Pilquimán, 2014; Marín \& Henríquez, 2015), donde convergen: 1) la resistencia, como una opción para salvaguardar y proteger territorios amenazados por intereses de grandes conglomerados económicos; 2) la revalorización de prácticas culturales, como un camino para mitigar los procesos de asimilación a la cultura nacional dominante; y 3) la generación de oportunidades, como una vía para reducir los problemas que sobreabundan en comunidades empobrecidas.

Respecto de este último punto, llama la atención que aun cuando Chile ha alcanzado un destacado lugar entre los países latinoamericanos en el Î́ndice de Desarrollo Humano (IDH) del Programa de las Naciones Unidas para el Desarrollo (PNUD), presenta importantes desigualdades entre las diversas regiones que componen el territorio nacional, resultados que son replicados a nivel comunal donde las comunas con mayor presencia de población rural muestran consistentemente menores niveles de logro en IDH, y dentro de éstas las que presentan una alta concentración de población indígena exhiben valores de IDH muy bajo y bajo respectivamente (MIDEPLAN-PNUD, 2000). Recientemente, el Informe de Desarrollo Social del Ministerio de Desarrollo Social (2015) confirma estos agudos contrastes desde el punto de vista de la incidencia de la pobreza entre las diferentes regiones y comunas, así como también que la incidencia de la pobreza en la zona rural

\footnotetext{
Si bien el Estado chileno en 2008 ha ratificado el Convenio nº 169 de la Organización Internacional del Trabajo (OIT), que enfatiza el derecho de los pueblos originarios a reconocer, proteger y desarrollar su patrimonio cultural, sus conocimientos tradicionales, a practicar y revitalizar sus tradiciones, usos y costumbres culturales, como también a mantener su integridad como pueblos distintos, se debe reconocer que la implementación de este convenio hasta la fecha en Chile ha sido muy insuficiente, especialmente en el caso del derecho de consulta que éste reconoce (véase Aylwin, 2003). Por otra parte, en Chile no existe un reconocimiento constitucional de los pueblos indígenas y sus derechos y un proyecto de reforma constitucional referido a esta materia descansa en el Congreso Nacional desde el año 2007 sin ningún progreso a la fecha. Un tímido avance legislativo han sido la Ley $\mathrm{n}^{\circ}$. 19.253 de 1993 sobre "fomento, protección y desarrollo de los indígenas” y la Ley $n^{\circ}$. 20.249, promulgada en 2008, que “crea los espacios marinos costeros de los pueblos originarios”, aunque ambas criticadas por tener diversas barreras institucionales para su implementación que no se condicen con el estándar del derecho internacional en materia de derechos de los pueblos indígenas (véase Aylwin, 2000; Zelada \& Park, 2015).
} 
(27,9\%) es significativamente mayor que en la urbana (12,4\%) afectando de manera especial a las personas pertenecientes a la etnia Mapuche.

Teniendo en cuenta que actualmente los pueblos indígenas demandan oportunidades reales de participación, decisión y acceso al conocimiento de un modo culturalmente pertinente (Velasco, 1999), se hace necesario respaldar iniciativas que junto con propiciar una reparación auténtica a comunidades que históricamente se han visto desfavorecidas por la marginación se proyecten hacia el futuro como estrategias de vida sustentable (Pilquimán \& Skewes, 2010).

En esta contribución exploramos en la trama de imaginarios generados en torno al turismo comunitario por parte de estas comunidades mapuche, entendiendo imaginario como "el conjunto de creencias, imágenes y valoraciones definidas sobre una actividad, un espacio, un periodo o una persona y/o sociedad en un momento dado" (Hiernaux, 2002: 8), una construcción social —al mismo tiempo individual y colectiva - en permanente cambio (Hiernaux, 2002), considerando que se presenta como una alternativa de desarrollo de acuerdo al propio discurso de las comunidades mapuche, sobre todo para aquellas que actualmente disponen de valiosos bienes paisajísticos en los territorios de refugio hacia donde antaño fueron desplazadas, tal es el caso de las comunidades mapuche ubicadas en la zona precordillerana de la comuna de Panguipulli (Región de Los Ríos).

Todo lo anterior para revelar elementos de información empírica acerca de cómo se desenvuelve el turismo en ciertas localidades donde predomina la presencia de población mapuche como también algunos aspectos que puedan contribuir a la construcción de un modelo de desarrollo turístico pertinente a los requerimientos más acuciantes de las realidades locales en territorios que, como es el caso de nuestro área objeto de estudio, progresivamente reflejan los efectos de la globalización.

\section{El contexto regional}

Las experiencias de turismo analizadas se ubican geográficamente en la comuna de Panguipulli, en la Región de Los Ríos, en el sur de Chile. Concretamente, en la zona precordillerana de esta comuna donde se ubican algunos de los denominados Lagos Araucanos presentes en el borde occidental de Los Andes entre la Novena y la Undécima Región cuya morfología ha experimentado en los últimos años una notoria transformación por el cambio de uso del suelo, desde bosque nativo a praderas para la industria lechera, plantaciones de árboles en monocultivo (Soto \& Lara, 2000) y desarrollo inmobiliario asociado al turismo (Constabel, 1997; Hidalgo \& Zunino, 2011).

Por la doble condición geográfica del territorio (rural-natural) ha interesado investigar en los ejes donde se inscribe la oferta turística en la zona precordillerana de la comuna de Panguipulli caracterizada por: 1) la belleza escénica lacustre, que ha permitido el ejercicio de diversas actividades al aire libre; 2) una abundante naturaleza, representada por el bosque templado y las reservas creadas para su protección; y 3) la presencia de aguas termales. Este conjunto de atributos naturales sintetizan y testimonian una parte de su riqueza patrimonial y además demuestran su potencial turístico (Mapa 1). 
Mapa 1. Ubicación geográfica de las áreas seleccionadas para los estudios de caso

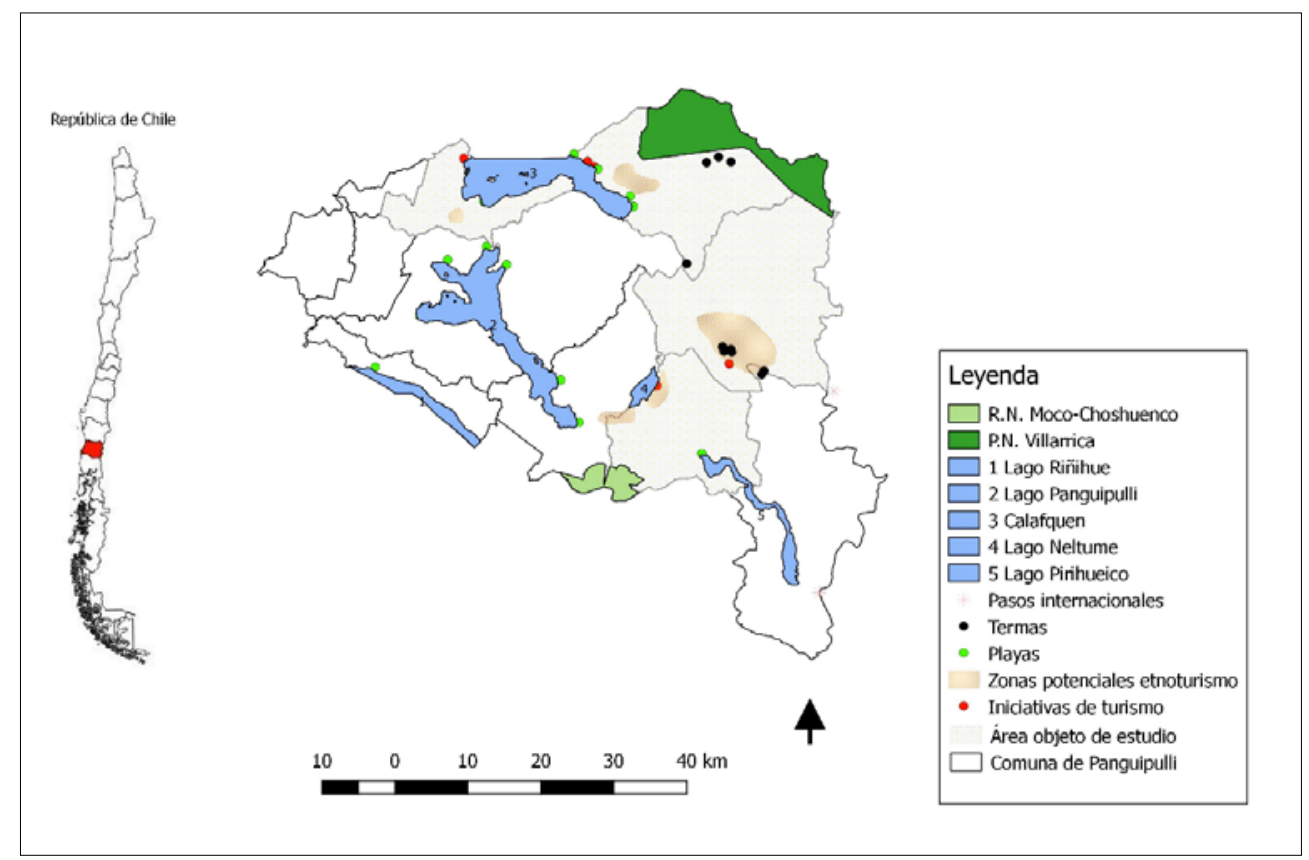

Fuente: Elaboración propia.

El atractivo paisajístico de esta zona además se complementa con la presencia de un sitio arqueológico denominado Marifilo. Es uno de los sitios arqueológicos más tempranos descubierto en la zona centro sur de Chile, cuyos hallazgos informan sobre la existencia de una ocupación constante desde el Arcaico Temprano hasta el período Formativo por parte de poblaciones adaptadas a los bosques templados que desarrollan una estrategia económica con un fuerte énfasis en la recolección y una marcada tradicionalidad (Adán et al., 2004). A este sitio se agrega en la ribera noreste del lago Calafquén los vestigios de una fortificación recientemente dada a conocer por una familia mapuche de la localidad que conformaría parte de un sistema defensivo utilizado por el pueblo mapuche en la denominada Guerra de Arauco $^{6}$.

Como contrapunto, hay que decir que corresponden a zonas ambientalmente frágiles que resguardan bienes patrimoniales — presencia de áreas silvestres protegidas de los Bosques Templados Lluviosos de los Andes Australes declarados Reserva de la Biosfera por la UNESCO_-, amenazadas tanto por la actividad maderera extractiva como por un turismo masivo que ejerce una fuerte presión sobre los recursos naturales donde se ha fomentado; además, el crecimiento del sector a través de una serie de programas estatales, que buscan diversificar la oferta turística local en sus componentes y contenidos, constituyéndose el patrimonio cultural de las comunidades mapuche en un activo añadido a la oferta turística local.

6 Con este nombre se conoce el enfrentamiento sostenido entre españoles y mapuche, que abarcaría todo el periodo colonial (siglos XVI a XVIII). 
Cuadro 1. Antecedentes demográficos y productivos del área objeto de estudio

\begin{tabular}{|c|c|c|c|c|c|c|c|c|c|}
\hline Localidad & 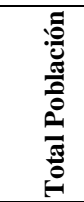 & 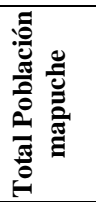 & 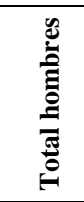 & 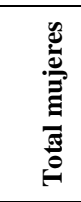 & 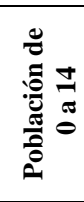 & 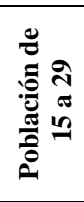 & 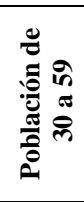 & 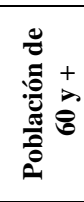 & 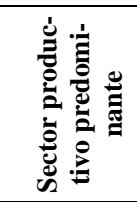 \\
\hline Coñaripe & 2.530 & 1.781 & 938 & 843 & 485 & 427 & 604 & 265 & Agricultura \\
\hline Liquiñe & 4.491 & 3.122 & 1.677 & 1.445 & 923 & 720 & 1.026 & 453 & Agricultura \\
\hline Neltume & 3.900 & 2.534 & 1.387 & 1.147 & 660 & 605 & 886 & 383 & Silvicultura \\
\hline Calafquén & 3.025 & 1.482 & 744 & 738 & 431 & 309 & 518 & 224 & Agricultura \\
\hline $\begin{array}{l}\text { Total área de estu- } \\
\text { dio }\end{array}$ & 13.946 & 8.919 & 4.746 & 4.173 & 2.499 & 2.061 & 3.034 & 1.325 & \\
\hline Total comuna & 33.273 & 10.231 & 5.327 & 4.904 & 2.910 & 2.323 & 3.513 & 1.485 & \\
\hline$\%$ en total comuna & 41,9 & 87,2 & 89,1 & 85,1 & 85,9 & 88,7 & 86,4 & 89,2 & \\
\hline
\end{tabular}

Fuente: Elaboración propia, en base al Censo de Población y Vivienda (INE, 2002 Versión Redatam).

Un elemento relevante a considerar en este territorio resulta ser, desde el punto de vista demográfico y etnográfico, la presencia importante de población mapuche (Cuadro 1). Predomina una población adulta que no muestra diferencias importantes según sexo; sin embargo, su distribución por edad refleja la existencia de una población joven menos numerosa respecto a la adulta, situación atribuible a las transformaciones ocurridas en el mundo rural que impulsa a la población joven a desplazarse hacia las grandes urbes en busca de nuevas oportunidades. Notable excepción ocurre en época estival, cuando los flujos turísticos se incrementan, siendo uno de los factores que ha motivado a las comunidades mapuche para vincularse a la actividad turística y mostrarse al "wingka turista" como una alternativa que permita mejorar su calidad de vida y fijar a los jóvenes en estas localidades (Mapa 1).

En lo específico, este artículo comprende el estudio de iniciativas de turismo diversas: 1) una Feria costumbrista a cargo de integrantes de una asociación de agricultores y artesanos constituida desde sus inicios por mujeres de origen mapuche que ofrecen diversos productos como gastronomía típica campesina y mapuche, artesanías en lana y madera, tejidos, productos de belleza orgánicos y plantas medicinales junto a una Comunidad indígena que brinda excursiones hacia áreas naturales, ambas ubicadas en Coñaripe; 2) una Agrupación de turismo ecológico y guías locales, compuesta por integrantes de varias comunidades rurales e indígenas en Liquiñe; 3) una Comunidad indígena organizadora de un evento de navegación ancestral y una feria de productos locales en Lago Neltume; y, por último, 4) una Comunidad indígena cuyos integrantes han puesto en valor su patrimonio natural dotándolo de servicios turísticos (alojamiento, alimentación y excursiones) en Calafquén.

\section{Metodología}

Para analizar la trama de imaginarios y experiencias generadas en torno al turismo por parte de comunidades indígenas mapuche en este trabajo de investigación se han elegido iniciativas de turismo ubicadas en las localidades de Coñaripe, Liquiñe, Neltume y Calafquén mencionadas previamente (Mapa 1). Esta selección co- 
rresponde al resultado de un análisis previo realizado desde una perspectiva más amplia en el marco del Proyecto denominado Nodo de Economía Solidaria y Turismo Comunitario en la Zona de Amortiguación del Parque Nacional Villarrica y Reserva Nacional Mocho Choshuenco y el Proyecto titulado Activación Turística del Patrimonio Cultural en Zonas Precordilleranas de las Regiones de los Ríos y Lagos.

El análisis empírico se ha fundamentado en un estudio de carácter exploratorio y descriptivo basado en el método del estudio de caso. En este contexto, un abordaje basado fundamentalmente en la implementación de una metodología de investigación cualitativa ha permitido acercarse a la temática investigada a través de testimonios orales mediante entrevistas grupales e individuales que en conjunto ascendieron a 40 personas, tratadas con posterioridad al análisis de contenido. Además, se han analizado testimonios recogidos en una serie de encuentros realizados en la escala local en el marco de los proyectos citados donde destaca la presencia de actores indígenas representantes de las experiencias estudiadas como también de otros representantes ligados al sector en sus distintas escalas. El trabajo de campo se ha realizado en distintos periodos entre los años 2013 y 2016.

Cuadro 2. Determinación de la muestra de estudio

\begin{tabular}{|c|c|c|c|}
\hline \multicolumn{3}{|l|}{ Universo } & 162.343 \\
\hline \multicolumn{3}{|c|}{ Error máximo aceptable } & 7 \\
\hline \multicolumn{3}{|c|}{ \% Nivel de confianza } & 90 \\
\hline \multicolumn{3}{|c|}{ Tamaño de la muestra } & 139 \\
\hline \multicolumn{4}{|c|}{ Muestra probabilística estratificada } \\
\hline Estratos & Total visitantes & Fracción constante & Total muestra \\
\hline Coñaripe & 82.383 & 0,0008562 & 71 \\
\hline Liquiñe & 53.306 & 0,0008562 & 46 \\
\hline Neltume & 12.115 & 0,0008562 & 10 \\
\hline Calafquén & 14.538 & 0,0008562 & 12 \\
\hline Total & 162.343 & & 139 \\
\hline
\end{tabular}

Fuente: Elaboración propia, en base a Encuesta Anual de Caracterización de la Demanda Turística de la Comuna de Panguipulli, año 2010

La perspectiva cualitativa que ha cimentado este estudio se ha complementado con la cuantitativa mediante la realización de un estudio sobre la demanda turística, utilizando la técnica de la encuesta, seleccionada por su idoneidad para obtener datos representativos de un universo de estudio. En función de los antecedentes proporcionados por el Departamento de Turismo de la Ilustre Municipalidad de Panguipulli se ha logrado precisar que 242.304 personas se desplazaron a la comuna de Panguipulli en el año 2010 —última cifra oficial disponible- por motivos recreacionales o de placer. De este total, el 67\% visitaron las localidades de Coñaripe, Liquiñe, Neltume y Calafquén, en cuyos territorios se emplazan las iniciativas de turismo en estudio (Mapa 1). Esta última cifra ha permitido definir el tamaño de la muestra para luego estratificarla. Para determinar cada estrato de la muestra se ha recurrido a los indicadores porcentuales sobre la distribución espacial de los visitantes en los territorios correspondientes a las localidades ya señaladas (Cuadro 2).

Disponiendo de estos antecedentes se ha identificado en el trabajo de campo las horas de mayor flujo de visitantes en las iniciativas de turismo en estudio. El levan- 
tamiento de datos se ha realizado en época estival a través de muestreo aleatorio en 2014.

Las principales conclusiones obtenidas del proceso investigador descripto fueron contrastadas con el ejercicio de la observación directa. En lo que sigue se expone parte de los resultados obtenidos.

\section{Análisis y resultados}

\subsection{Expansión turística en territorios conflictivos}

Las experiencias de turismo analizadas se ubican en una antigua área de refugio a la que accedieron las comunidades mapuche de la zona (Skewes et al., 2012) después de la llamada "Pacificación de la Araucanía". Estas áreas se constituyeron en espacios de aislamiento y subsistencia de la población mapuche (Bengoa, 1996) conforme a los avances de la colonización y la expansión capitalista; sin embargo, desde la década de 1970 se han visto amenazadas por la penetración del modelo territorial neoliberal (Toledo, 2006).

En el caso del turismo, debido al incremento de las migraciones turísticas en especial de origen nacional ${ }^{7}$. Con el nacimiento del "Destino Turístico Siete Lagos" el paisaje de las cuencas lacustres se ofrece a los visitantes, así las aguas han adquirido un valor escénico produciéndose un progresivo distanciamiento entre las propiedades del interior y las aledañas a los cursos de agua, las que han sido vendidas por parte de la población local o entregadas en comodato $^{8}$ a emprendedores foráneos (Skewes et al., 2012), hecho que ha favorecido el asiento de una oferta turística en crecimiento causante de una serie de impactos que han afectado de manera especial a la población mapuche, que, aun cuando reconoce los beneficios económicos obtenidos de la actividad turística es consciente de las tensiones y conflictos generados por la misma al colonizar y competir por la ocupación y uso del territorio, generando un frágil equilibrio marcado por conflictos emergentes debido a la existencia de dos representaciones simbólicas y discursivas del territorio, confrontadas político y semánticamente (Toledo, 2006).

En la cosmovisión indígena mapuche del bienestar humano la tierra y el territorio están profunda e indisolublemente vinculados a su historia y cultura, por ende, a su pervivencia como pueblo (Tricot, 2009). El territorio es un espacio constitutivo de su cultura e identidad, donde coexisten la cosmovisión, la comunidad de la sangre o Küpan, la identidad territorial o Tüwun, las tradiciones ancestrales, la memoria cultural, el andamiaje social, el idioma (Tricot, 2009). Pero con la economía neoliberal, el territorio se ha puesto al servicio de actividades globales; en el caso del turismo, al convertirlo en un escenario construido estratégicamente para atender a las necesidades de la demanda turística (Carvalho \& Guzmán, 2011), su imple-

Los datos del estudio de la demanda indican que la mayor parte de las personas que visitan el área objeto de estudio provienen de Chile, principalmente de la ciudad de Santiago (50\%) y centros urbanos de las regiones de La Araucanía y Los Ríos, de las ciudades de Temuco (10,8\%) y Valdivia (7,2\%) respectivamente.

8 El comodato o préstamo de uso es un contrato en que una de las partes entrega a la otra gratuitamente una especie, mueble o raíz, para que haga uso de ella, y con cargo de restituir la misma especie después de terminado el uso (Artículo 2174 del Código Civil). 
mentación desde esta lógica ha olvidado la realidad y aspiraciones de las comunidades mapuche al no considerar que las opciones turísticas predeterminadas pueden ocasionar una mercantilización del espacio, (Pilquimán \& Skewes, 2010).

Figura 1. Espacio recuperado por parte del lof Traitraiko destinado al turismo

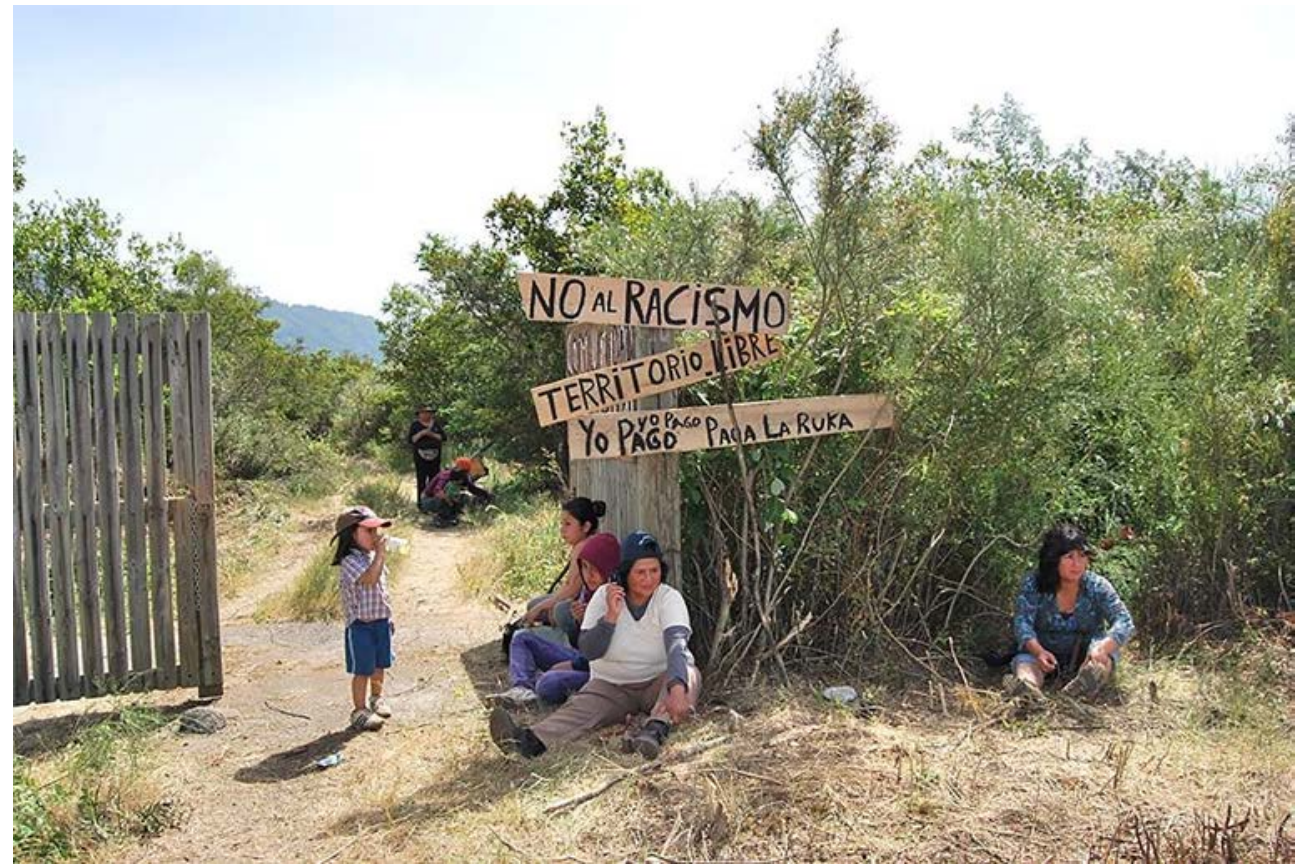

Fuente: Fotografía realizada por Marisela Pilquimán.

En el caso del paisaje ribereño del Lago Calafquén, la eficacia colonizadora del turismo se aprecia en la privatización de los mejores espacios destinados a la construcción de segundas residencias y en el uso recreativo intensivo de las playas por parte de los visitantes en desmedro de las posibilidades de uso de las comunidades mapuche, situación que ha detonado en varias disputas, la más reciente con el Estado chileno y particulares tras un proceso de recuperación territorial sobre un terreno ubicado en la ribera de este lago, que las comunidades ancestrales reivindican como propio en virtud de la posesión de un antiguo Título de Merced. Cabe destacar que con esta recuperación las comunidades han logrado vincularse al turismo, teniendo presente que previamente su participación se limitaba en muchos casos a la comercialización de algunos productos típicos, pese a estar ubicados en un espacio que concentra a un contingente importante de atractivos turísticos.

En sus propias palabras, el sentimiento que estas comunidades experimentan sería el siguiente: "Nuestra playa reivindicada había sido usurpada... ahora se utiliza comunitariamente con la comunidad y el lof ${ }^{9}$ de Traitraiko para hacer turismo”, explica I. C. (2015) (Figura 1).

9 Lof: corresponde a una agrupación social que involucra “a un número importante de familias las cuales poseen una relación de consanguineidad con el lonko (jefe), pero cada una de dichas familias conserva su independencia territorial” (Bengoa, 2004: 276). 
En el caso de Lago Neltume, a los usos recreativos ligados al lago se une la aparición de otras posibles ocupaciones productivas de los potenciales recursos lacustres, como, por ejemplo, los hidroenergéticos. Esto crea un clima de incertidumbre y resistencia, protagonizado por las comunidades mapuche enfrentadas a las presiones de una gran empresa trasnacional vinculada a la puesta en marcha de mega proyectos energéticos que pretende cambiar el curso de las aguas de la cuenca lacustre para generar energía, y, en consecuencia, modificar las características paisajísticas del lugar (Skewes et al., 2012).

Figura 2. Expedición hacia el Glaciar Pichillanchue organizado por comunidades mapuche aledañas al Parque Nacional Villarrica

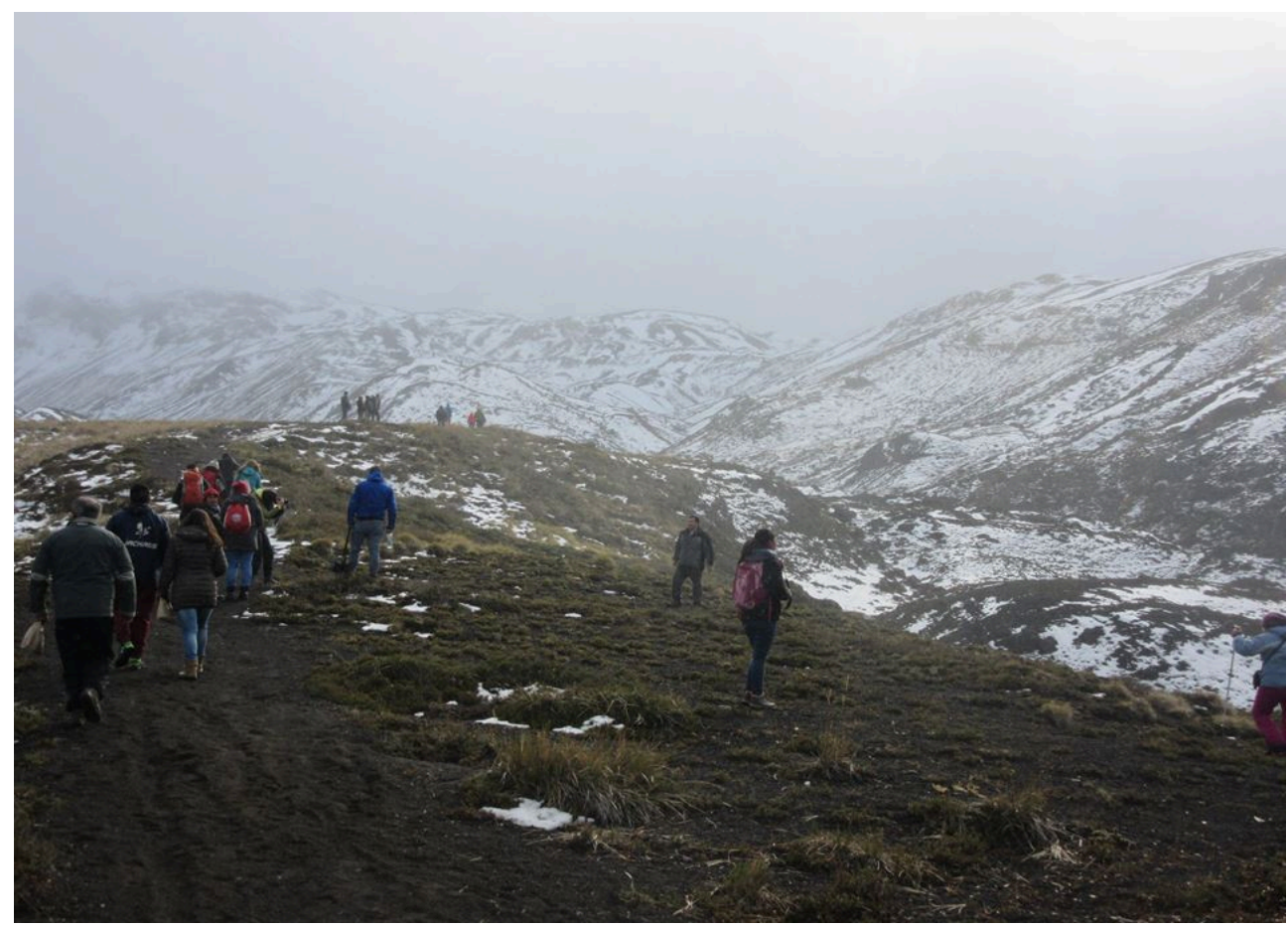

Fuente: Fotografía realizada por Angélica Chincolef.

La preocupación radica en que una modificación territorial importante afectaría a su patrimonio cultural inundando espacios sagrados de alta significación donde se recrea su cultura y cosmovisión además de lesionar el desarrollo de sus proyectos turísticos al constatar, a través del análisis de la demanda realizado en el área de estudio, que los elementos del paisaje que concitan un mayor entusiasmo con fines recreativos y/o estéticos son: los cuerpos de agua, el relieve (montañas y volcanes) y la vegetación, cuya trasformación condicionaría el regreso de 77\% de los visitantes.

Respecto a las áreas naturales, los usos recreativos especialmente del Parque Nacional Villarrica son realizados mayoritariamente por operadores turísticos exógenos - aunque hay significativas excepciones (Figura 2) - que desconocen la existencia de espacios en este Parque Nacional destinados a prácticas religiosas, 
espirituales y productivas de las comunidades aledañas, por ejemplo en torno al volcán Villarrica (Ruka Pillán o casa de espíritus), el uso de las zonas más altas como veraneadas ${ }^{10}$ para la recolección de piñones ${ }^{11}$ y el pastaje y la existencia de una ruka o refugios para utilizar en dichas veraneadas (Aylwin, 2008); situación constantemente criticada por las mismas: "La gente que hace trekking, cabalgatas, la que recorre estos lugares no es mapuche, pasan por territorio mapuche, pero nadie sabe que este territorio es indígena”, expresa J. C. (2013).

Todo lo anterior origina un panorama complejo, más aun considerando la actual voluntad estatal de implementar planes de desarrollo turístico, que contemplan licitaciones de áreas circunscritas a este Parque Nacional, territorio que desde el imaginario del pueblo mapuche corresponden a su dominio ancestrales y, por tanto, forman parte de sus reivindicaciones ${ }^{12}$ (Aylwin, 2008).

Por otra parte, las presiones desmedidas sobre los recursos turísticos del territorio ancestral mapuche derivan tanto de la intensificación de la actividad económica como de la creciente concentración poblacional estacional en los núcleos urbanos de Coñaripe y Licán Ray, en especial. Esta situación se refleja en diversos aspectos: la llegada de un contingente de comerciantes ambulantes, cuya presencia en el territorio ocasiona conflictos con la población local; la generación de residuos y ruidos; el aumento del número de vehículos terrestres y recreativos fluviales, etc. Todas estas externalidades ambientales negativas son fiel testimonio del carácter más bien inorgánico, antes que planificado, asumido por la actividad turística en el área de estudio.

\subsection{Entre turismo masivo y turismo comunitario}

A partir de las reflexiones realizadas por parte de los representantes de las experiencias estudiadas se siguen dos diagnósticos o interpretaciones acerca de la implantación del turismo en el área de referencia empírica de este estudio.

La primera se asocia a la visión dominante del mismo en cuanto a motor dinamizador de un espacio rural integrado en un sistema enraizado en intereses económicos al servicio de actividades globales. Desde este punto de vista, la nueva ruralidad se asocia a actividades productivas como los "monocultivos" forestales, la agricultura de exportación e incluso al turismo masivo. A estos megaproyectos económicos se resisten las comunidades mapuche, pues les obliga, por una parte, a abandonar sus actividades productivas tradicionales $\mathrm{y}$, por otra, tiende a homogeneizar sus espacios ancestrales. De esta forma, el proceso de ruptura con el modelo económico neoliberal que tiende al crecimiento constante y genera presión sobre los territorios vía la especulación inmobiliaria (Marín \& Henríquez, 2015) visto

10 Las veraneadas corresponden a una práctica asociada a la recolección del piñón realizada por familias Mapuche en las zonas precordilleras de la Cordillera de Los Andes.

11 Piñon: fruta proveniente del árbol Araucaria Araucana que forma parte de la dieta de familias Mapuche en zonas precordilleranas. Comúnmente es utilizado para la confección de harina o en otras preparaciones.

12 El territorio en que se ubica el Parque Nacional Villarrica es un área cuya administración desde antiguo ha sido ejercida por los lonko o cabezas de familia. Ello da cuenta de la existencia de un sistema de posesión, propiedad y control ancestral sobre dichos espacios (Aylwin, 2008). Junto a lo anterior, varias comunidades cuentan con títulos de propiedad otorgados por el Estado y que se sobreponen a las tierras que hoy el fisco pretende inscribir para sí. Véase, por ejemplo, Causal rol C-3862-2013, caratulada “Fisco con Caripán”, del $1^{\circ}$. Juzgado Civil de Valdivia. 
desde la mirada occidental o "winka", como lo denominan los mapuche, es evidente. En el caso concreto del turismo, vinculado al turismo masivo, a su juicio, fomenta la colonización de sus espacios, el individualismo, la concentración de la propiedad y el desplazamiento de la población local hacia otros territorios.

Frente a esta perspectiva surge una segunda interpretación del turismo en torno a planes de vida vinculados al Küme Mongen, Buen Vivir, en mapuzugún, que al igual que en los casos del Suma Qamaña aymara o Suma Kawsay quechua, podría considerarse como un nuevo concepto que actualiza los principios filosóficos y epistemológicos de la cosmovisión mapuche, fundamentados en el concepto ancestral del Kimün, o sabiduría, el cual incluye los conocimientos sobre todas las dimensiones territoriales tanto materiales como inmateriales (Viera, 2013).

Nosotros hablamos del Küme Mongen (buen vivir), de generar las condiciones para que nuestras familias y las familias que habitan en el territorio logren mejorar su calidad de vida en términos económicos, culturales y espirituales en armonía con las diferentes especies que habitan el territorio, dice S. M. (2014).

Desde esta perspectiva, el turismo comunitario se posiciona en el imaginario colectivo como una estrategia incipiente para la revitalización cultural, pero también para la defensa territorial:

Nosotros dijimos (...) vamos a hacer un turismo distinto, que tenga otros valores (...) Nos pusimos el desafío de trabajar el tema del turismo comunitario: otra mirada, otra forma de recibir a la gente, de compartir con ellos, de contarles la historia, de que también ellos sean, se sientan, como un poco parte de este pedacito de espacio (M. L., 2015). Y M. H. (2014) agrega: Al dar a conocer esta localidad queremos rescatar la historia (...) aquellas características sobre la forma de vida que nuestros antepasados nos legaron, o sea, sus aspectos culturales (formas de vivir) ya sea la agricultura, entorno familiar y social, etc.

\subsection{Encuentros y desencuentros entre comunidades anfitrionas y visitantes}

En la actualidad estas experiencias de turismo despiertan el interés entre visitantes con perfiles sociodemográficos diversos, pero semejante respecto a las principales motivaciones para desplazarse hacia estas localidades. El 61,2\% son hombres y el 38,8\% mujeres, con edades que fluctúan entre los 18 y 65 años, agrupándose mayormente en el segmento de adultos jóvenes (30 a 44 años), adultos (45 a 64 años) y jóvenes (18 a 29 años). La mayor parte ha cursado estudios universitarios (48,2\%) y técnicos $(27,3 \%)$.

Predomina una demanda de origen nacional dividida en tres grandes grupos. El primero procedente de la ciudad de Santiago (50\%). El segundo procede de centros urbanos de la Región de la Araucanía y Los Ríos, de las ciudades de Temuco $(10,8 \%)$ y Valdivia (7,2\%) respectivamente y de otras localidades cercanas al lugar de destino (2,2\%). El tercer grupo reúne a visitantes de ciudades de otras regiones del país (30,2\%). Cabe destacar que la presencia de visitantes de origen extrajero es reducida $(0,7 \%)$.

Al consultar a los visitantes acerca de sus motivaciones para desplazarse al área de estudio han sido enunciadas como las principales: el disfrutar de la naturaleza, 
las playas y las aguas termales. Sin embargo, otra especial motivación que manifiestan muchos visitantes es el interés por "conocer la cultural rural y mapuche”.

Entre quienes expresan su interés por conocer in situ los atributos de la cultura mapuche surgen diversas apreciaciones al momento de experimentar este tipo de experiencias relacionadas a su idealización sobre lo que entienden por auténtico. Ello responde, en primer lugar, a un catálogo de características preestablecidas en sus imaginarios, mediante las cuales definen a la población mapuche antes de producirse la interacción turística, incluyendo la existencia de una serie de estereotipos y prejuicios sustentados por parte de la sociedad dominante y, en segundo lugar, al grado de pauperización presentados por los propios servicios turísticos.

Cabe destacar que "en términos de relaciones interétnicas con los indígenas, Chile exhibe una realidad contradictoria, desde su discurso social y público los miembros de la sociedad dominante se muestran abiertos y tolerantes respecto de los indígenas" (Merino, 2007: 606). Sin embargo, en términos de relaciones interpersonales e interacción cotidiana la actitud generalizada de la sociedad no indígena tiende a ser distante, desconfiada y prejuiciada respecto de los indígenas, especialmente respecto de los mapuche en tanto grupo indígena mayoritario en Chile (Mellor et al., 2009).

No obstante, como ya señalamos, las personas que visitan el área objeto de estudio muestran interés por este tipo de experiencias y realizan una serie de recomendaciones destinadas a mitigar las deficiencias por ellos/ellas detectadas. Estas sugerencias varían desde el mejoramiento de aspectos ligados a la propia iniciativa de turismo, el ensanchamiento de la oferta de actividades recreativas relacionadas con la naturaleza y cultura, el mejoramiento del equipamiento y cuidado del entorno, hasta la intensificación de los esfuerzos publicitarios para darlas a conocer.

Desde la perspectiva de las comunidades anfitrionas este tipo de encuentros ha favorecido la generación de ingresos complementarios destinados a alentar sus desanimadas economías; pero su disposición favorable hacia la actividad turística no significa que estén dispuestas a compartir rasgos más íntimos de su cultura, aparte de los que sustentan los servicios ofrecidos en los espacios destinados a las interacciones turísticas.

Asimismo, aunque de naturaleza ocasional y en algunos casos hasta fugaz, la interacción con los visitantes, aparte de representar una fuente de ingresos, se ha constituido, desde su punto de vista, en un medio de promoción y defensa de su territorio y cultura. Igualmente, el turismo se posiciona, desde esta perspectiva, como una alternativa para reconstruir prácticas tradicionales pérdidas o postergadas como resultado de los procesos de asimilación a la sociedad mayoritaria, aunque sin caer en el extremo de transformar su cultura en un espectáculo para satisfacer las expectativas del visitante. Esta postura obedece ante todo a que las comunidades mapuche buscan visibilizar sus modos de vida para divulgar cómo su relación con la naturaleza ha posibilitado la conservación de la biodiversidad local mediante la puesta en valor de ciertas prácticas tradicionales ligadas a los usos sustentables del territorio con el objeto de constituirlas en la base de sus iniciativas de turismo. 


\section{Conclusiones}

Los casos de estudio expuestos en este artículo invitan a reflexionar sobre la implantación del turismo en zonas precordilleranas de la comuna de Panguipulli, en la Región de Los Ríos, sur de Chile, protagonistas de un crecimiento turístico exponencial en los últimos años, como consecuencia del incremento de las migraciones turísticas, especialmente en época estival. La discusión pone de relieve no sólo los beneficios económicos de la actividad turística, sino también la trama de tensiones y conflictos emergentes generados sobre espacios habitados predominantemente por población mapuche. Los grupos mapuche reconocen los beneficios derivados del asiento de actividad turística en los territorios que habitan, pero, igualmente manifiestan los problemas ocasionados por su práctica, al competir por la ocupación y uso del espacio con los modos de vida y actividades económicas tradicionales.

Si bien las experiencias analizadas reflejan que el turismo comunitario ha resultado especialmente atractivo para integrantes de comunidades mapuche que apuestan por un turismo de baja escala como una fórmula para mitigar los múltiples problemas que enfrentan. Estas colectividades originarias reconocen, además, que la puesta en valor de su patrimonio se convierte en un medio de resistencia y reivindicación política respecto de la soberanía sobre los recursos existentes en sus territorios; sin embargo, también revelan los múltiples desafíos a los cuales se deben enfrentar para sobrevivir en el sistema turístico imperante e impuesto bajo las lógicas capitalistas de mercado neoliberales.

En este contexto, resultaría recomendable propender a políticas públicas que subsidien la construcción de espacios de encuentro destinados a favorecer el establecimiento de relaciones de tipo intercultural a través del turismo, que realcen la propia especificidad de estas comunidades, su forma de vida y cultura, y, a través de las mismas, se contribuya al reconocimiento y protección de su patrimonio. En consecuencia, de cómo se formulen estas políticas públicas, en concordancia con las necesidades locales, la participación de la comunidad local y las estrategias que se adopten para minimizar los impactos negativos sobre el patrimonio, los recursos naturales y las formas de vida locales dependerá la satisfacción de las necesidades y aspiraciones de estas comunidades vinculadas al turismo, o si simplemente se contribuye a fomentar la mercantilización de su patrimonio para satisfacer las tendencias de la demanda turística.

Se debe, en consecuencia, avanzar en una propuesta en la cual el turismo trascienda a una simple estrategia de localización espontánea de nuevos procesos productivos en el espacio rural fortaleciendo los esfuerzos realizados por parte de estas comunidades en favor de una propuesta de turismo que contribuya a mantener vigente el conocimiento del territorio, la sabiduría y la tradición mapuche situación que implica previamente la comprensión profunda sobre cuáles han sido los acontecimientos históricos que han moldeado su realidad, pues sólo a partir del manejo de este conocimiento se podrán diseñar estrategias turísticas que respondan de manera más efectiva a las necesidades que emanan de las propias comunidades. 


\section{Bibliografía}

Adán, Leonor; Mera, Rodrigo; Becerra, Marcela \& Godoy, Marcelo (2004) “Ocupación arcaica en territorios boscosos y lacustres de la región precordillerana andina del centrosur de Chile: el sitio Marifilo-1 de la localidad de Pucura”. Chungara. Revista de Antropología Chilena, núm. 36, 1121-1136.

Aylwin, José (2008) “¿De quién es el Parque Nacional Villarrica?”. Diario La Nación. [URL:

<http://www.lanacion.cl/noticias/site/artic/20081206/pags/20081206213705.html>. Consultado el 19 de mayo 2014].

Aylwin, José (2003) Pueblos indígenas en el Derecho Internacional y Comparado. [URL: $<$ http://200.10.23.169/trabajados/Jos\%E9\%20Aylwin.pdf>. Consultado el 22 de mayo 2015].

Aylwin, José (2000) "Los conflictos en el territorio mapuche: antecedentes y perspectivas”. Revista Perspectivas, vol. 3, núm. 2, 277-300.

Bengoa, José (2004) La memoria olvidada. Historia de los pueblos indígenas de Chile. Santiago de Chile: Bicentenario.

Bengoa, José (1996) "Población, familia y migración mapuche. Los impactos de la modernización en la sociedad mapuche. 1982-1995”. Revista Pentukun, núm. 6, 9-28.

Bengoa, José (1985) Historia del pueblo mapuche. Santiago de Chile: SUR.

Bohórquez, María Fernanda; Jurado, Claudia \& Duarte, Sonia (2015) “Turismo comunitario en la cuenca del Río Tunjuelo en Bogotá: Hacia un compromiso con la sostenibilidad desde la apropiación del territorio”, en M. Corrales \& K. Pino (coords.) El turismo y su relación con la comunidad. El desafío de la sustentabilidad. Santiago de Chile: Universidad Central, 71-84.

Bustos, Alejandro (2005) "Hacia un turismo intercultural: el caso atacameño”. Revista LIDER, núm. 13, 133-150.

Carvalho, Karoliny \& Guzmán, Sócrates (2011) “El turismo en la dinámica territorial. ¿Lógica global, desarrollo local?”. Estudios y Perspectivas en Turismo, vol. 20, núm. 2, 441-46.

Coca, Agustín (2012) “Turismo comunitario como estrategia de resistencia en el Alto Napo ecuatoriano”, en M. Valcuende (ed.) Amazonía, viajeros, turistas y poblaciones indígenas. Sevilla: Colección PASOS edita $n^{\circ}$. 6, 261-292.

Comisión Verdad Histórica y Nuevo Trato con los Pueblos Indígenas (2008) Informe de la Comisión Verdad Histórica y Nuevo Trato con los Pueblos Indígenas. [URL: <http://www.memoriachilena.cl/602/articles-122901_recurso_2.pdf>. Consultado el 25 de mayo 2015].

Constabel, Silvia (1997) "Propuestas para el ordenamiento turístico comunal: comunas de Futrono y Lago Ranco, Región de Los Lagos, Chile”. Gestión Turística, núm. 1, 100113.

Cox, José Ricardo (2012) "El turismo en la Amazonía Boliviana, a la luz de las políticas públicas y la emergencia de los pueblos indígenas”, en M. Valcuende (ed.) Amazonía, viajeros, turistas y poblaciones indígenas. Sevilla: Colección PASOS edita $n^{\circ}$. 6, 329352.

Grimm, Isabel. \& Sampaio, Carlos (2011) "Turismo de base comunitária: convivencialidade e conservação ambiental”. Revista Brasileira de Ciências Ambientais, núm. 19, 5768. 
Guzmán-López, Tomás; Borges, Osvaldo \& Castillos Canalejo, A. (2011) “Desarrollo económico local y turismo comunitario en países en vías de desarrollo. Un estudio de caso”. Revista de Ciencias Sociales, vol. XVII, núm. 3, 432-446.

Hidalgo, Rodrigo \& Zunino, Hugo (2011) "Negocios inmobiliarios en centros turísticos de montaña y nuevos modos de vida: El papel de los migrantes de amenidad existenciales en la Comuna de Pucón-Chile”. Estudios y perspectivas en turismo, vol. 20, núm. 2, 307-326.

Hiernaux, Daniel (2002) “Turismo e Imaginarios”. Cuaderno de Ciencias Sociales, núm. 123, 7-36.

Instituto Nacional de Estadística (INE) (2002) Hojas Informativas Estadísticas Sociales Pueblos Indígenas en Chile, Censo 2002 [URL: $<$ http://www.ine.cl/canales/chile_estadistico/estadisticas_sociales_culturales/etnias/pdf/i nfo_etniascenso2002.pdf>. Consultado el 4 de enero 2016].

Marimán, Pablo (2006) "Los Mapuches antes de la conquista militar chileno-argentina”, en P. Mariman, S. Caniuqueo, J. Millalén \& R. Levil (eds.) ¡Escucha, winka! Cuatro ensayos de Historia Nacional Mapuche y un epílogo sobre el futuro. Santiago de Chile: Lom Ediciones, 53-127.

Marín, Maritza \& Henríquez, Christian (2015) “Turismo de base comunitaria frente a conflictos etno-políticos y socio-ambientales en comunidades mapuche del sur de Chile”, en A. Pinol (ed.) Democracia versus neoliberalismo. 25 años de neoliberalismo en Chile. Santiago de Chile: CLACSO, 304-323.

Mellor, David; Merino, María Eugenia; Saiz, José \& Quilaqueo, Daniel (2009) “Emotional reactions, coping and long-term consequences of perceived discrimination among the Mapuche of Chile”. Journal of Community \& Applied Social Psychology, vol. 19, núm. 6, 473-491.

Merino, María Eugenia (2007) "El discurso de la discriminación percibida en Mapuches de Chile”. Discurso \& Sociedad, vol. 1, núm. 4, 604-622.

MIDEPLAN-PNUD (2000) Desarrollo humano en las comunas de Chile [URL: $<$ http://desarrollohumano.cl/idh/download/Desarrollofinal.pdf $>$. Consultado el $10 \mathrm{de}$ enero 2016].

Ministerio de Desarrollo Social (2015) Informe de Desarrollo Social, 2015 [URL: $<$ http://www.ministeriodesarrollosocial.gob.cl/pdf/upload/Libro_IDS_2015_final.pdf>. Consultado el 11 de enero 2016].

Organización Mundial del Turismo (OMT) (2015) Panorama OMT del turismo internacional, edición 2015 [URL: <http://www.e-unwto.org/doi/pdf/10.18111/9789284416875>. Consultado el 4 de enero 2016].

Pilquimán, Marisela (2014) “Turismo comunitario como estrategia de supervivencia y resistencia de comunidades mapuche en el sur de Chile”, en J. Carpio, F. Cebrián, M. Mínguez \& O. González (eds.) Revisando paradigmas y creando alianzas. Madrid: Asociación de Geógrafos Españoles, 1469-1482.

Pilquimán, Marisela \& Skewes, Juan Carlos (2010) "La comunidad indígena de ChoroyTraiguén, Provincia de Osorno, y sus proyectos de desarrollo”. Revista Líder, núm. 16,105-124.

Ruiz, Esteban \& Solís, Doris [coords.] (2007) Turismo comunitario en Ecuador: desarrollo y sostenibilidad social. Quito: Abya Yala / Universidad de Cuenca. 
Sampaio, Carlos \& Zamigan, G. (2012) "Estudo da demanda turística: experiência de turismo comunitário da Microbacia do Rio Sagrado, Morretes (PR)”. CULTUR: Revista de Cultura e Turismo, núm. 1, 25-39

Sepúlveda, C. (2001) La economía del turismo en Chile, políticas públicas y recursos humanos. Santiago (Chile): CEPAL.

Schaerer, Jorge \& Dirven, Martine (2001) El turismo rural en Chile. Experiencias de agroturismo en las Regiones del Maule, La Araucanía y Los Lagos. Santiago (Chile): CEPAL-ECLAC.

Skewes, Juan Carlos; Solari, María Eugenia; Guerra, Debbie \& Jalabert, Daniela (2012) "Los paisajes del agua: naturaleza e identidad en la cuenca del río Valdivia”. Chungara. Revista de Antropología Chilena, vol. 44, núm. 2, 299-312.

Solís, Doris (2007) "De la resistencia a la sostenibilidad: el proceso histórico del turismo comunitario en Ecuador y sus retos actuales”, en E. Ruiz \& D. Solís (coords.) Turismo comunitario en Ecuador: desarrollo y sostenibilidad social. Quito: Abya Yala / Universidad de Cuenca, 29-50.

Soto, Doris \& Lara, Antonio (2000) “Servicios Ecosistémicos de los Bosques Nativos”, en R. Primack, R. Rozzi, P. Feinsinger, R. Dirzo \& F. Mazzardo (eds.) Fundamentos de Conservación Biológica: Perspectivas Latinoamericanas. México D.F.: Fondo de la Cultura Económica, 295-297.

Toledo, Víctor (2006) Pueblo Mapuche, Derechos Colectivos y Territorio: Desafíos para la sustentabilidad democrática. Santiago (Chile): LOM Ediciones.

Tricot, Tito (2009) "El nuevo movimiento mapuche: hacia la (re)construcción del mundo y país mapuche”. POLIS, Revista de la Universidad Bolivariana, vol. 8, núm. 24, 175-19.

Velasco, Luisa (1999) "Lo «etno» del Desarrollo: una mirada a las estrategias y propuestas de desarrollo indígena”. Revista Mad, núm. 1 [URL: <http://www.revistamad.uchile.cl/index.php/RMAD/article/view/14864/15281>. Consultado el 16 de junio de 2016].

Viera, Patricia (2013) "Küme mongen: posibilidades y limitaciones de una pro-puesta de desarrollo generado desde el pueblo mapuche”. Memoria del Foro Bienal Iberoamericano de Estudios del Desarrollo. Santiago (Chile), Universidad de Santiago de Chile. [URL:

$<$ http://riedesarrollo.org/memorias/2013/mesas/mesa2/2.III.2\%20Patricia\%20Viera_Ku e\%20mongen\%20posibilidades\%20y\%20limitaciones\%20de\%20una\%20propuesta.pdf $>$. Consultado el 20 de junio de 2016].

Zelada, Sara \& Park, James (2015) “Análisis crítico de la Ley Lafkenche ( $\mathrm{N}^{\circ}$. 20.249): El complejo contexto ideológico, jurídico, administrativo y social que dificulta su aplicación”. Universum (Talca), vol. 28, núm 4, 47-72. 